\title{
MULTICULTURALISME ET GENRE : ENTRE SPHÈRES PUBLIQUE ET PRIVÉE
}

\author{
Anouk Guiné
}

L'Harmattan I Cahiers du Genre

$2005 / 1-n^{\circ} 38$

pages 191 à 211

ISSN 1298-6046

Article disponible en ligne à l'adresse:

http://www.cairn.info/revue-cahiers-du-genre-2005-1-page-191.htm

Pour citer cet article :

Guiné Anouk, « Multiculturalisme et genre : entre sphères publique et privée »,

Cahiers du Genre, 2005/1 n³8, p. 191-211. DOI : 10.3917/cdge.038.0191

Distribution électronique Cairn.info pour L'Harmattan.

(C) L'Harmattan. Tous droits réservés pour tous pays.

La reproduction ou représentation de cet article, notamment par photocopie, n'est autorisée que dans les limites des conditions générales d'utilisation du site ou, le cas échéant, des conditions générales de la licence souscrite par votre établissement. Toute autre reproduction ou représentation, en tout ou partie, sous quelque forme et de quelque manière que ce soit, est interdite sauf accord préalable et écrit de l'éditeur, en dehors des cas prévus par la législation en vigueur en France. II est précisé que son stockage dans une base de données est également interdit. 


\title{
Multiculturalisme et genre : entre sphères publique et privée
}

\begin{abstract}
Anouk Guiné
Résumé

Les tensions entre universalisme et particularisme posent la question des limites de la différence culturelle et du droit des membres d'un groupe ethnique à cette différence. Jusqu'où peut-on aller dans la reconnaissance et la protection des spécificités culturelles, donc des droits culturels du groupe ? Le droit à la reconnaissance est-il souhaitable dès lors qu'il restreint la liberté d'une partie des membres du groupe ? Certains courants de pensée britanniques sur la diversité culturelle relèguent celle-ci à la sphère privée, d'autres l'associent à la sphère publique. Dans les deux cas, la vision du groupe est homogénéisante, seule l'identité collective est prise en compte, au détriment du droit des individus et en particulier des droits individuels des femmes. À travers le cas de l'excision pratiquée en Grande-Bretagne dans l'immigration somalienne, il s'agit de montrer comment la compatibilité entre culture des spécificités et idéaux égalitaires relève de la responsabilité de l'État, et passe avant tout par la protection des individus lorsqu'une pratique coutumière les met en danger.
\end{abstract}

MULTICULTURALISME — GRANDE-BRETAGNE — PUBLIC — PRIVÉ — EXCISION « RACE » — ETHNICITÉ — POLITIQUES MIGRATOIRES — SOMALIE

Le multiculturalisme et l'articulation entre privé et public comptent parmi les principales questions qui se posent aux sociétés dites démocratiques. Elles sont liées entre elles dans la mesure où, en cherchant à rendre compatibles culture des spécificités et culture civique, les théories classiques du multi- 
culturalisme - à l'instar des conceptions modernes de l'État de droit - envisagent les sphères publique et privée selon un principe de séparation et d'opposition.

Je me propose d'abord de montrer les principaux traits de l'évolution de la conceptualisation de ces deux sphères à partir de la théorie politique des courants dominants et des recherches féministes. Ensuite, je croiserai les conceptions traditionnelles du multiculturalisme britannique et des relations privé / public selon la perspective de genre - à travers le cas d'une pratique coutumière : l'excision, pratiquée aujourd'hui dans certaines communautés africaines en Grande-Bretagne.

Le genre est une catégorie d'analyse que je définis comme un système d'inégalités fondé sur la construction sociale des différences biologiques de sexe, fonctionnant principalement au détriment des femmes. Une analyse en termes de genre permet de montrer l'impact des théories classiques du multiculturalisme et de la dichotomie privé / public sur les droits individuels des femmes dans le contexte britannique actuel, notamment lorsque celles-ci sont privées de liberté au nom de la tradition.

\section{Genre et définitions des sphères privée et publique}

Depuis la rationalisation de la société au XIX ${ }^{\mathrm{e}}$ siècle, le privé et le public sont traditionnellement pensés comme une distinction entre, d'un côté, vie personnelle, individuelle, passions, reproduction et particularismes culturels, et, de l'autre, vie politique, collective, raison, production et application du droit dans une perspective universelle. Seule la famille est vue comme intermédiaire et dénominateur commun entre ces deux sphères (Perrot 1998, p. 386). C'est également au XIX ${ }^{\mathrm{e}}$ siècle que le principe de sexuation des espaces légitime la théorie des sphères publique et privée. Construite depuis le XVIII ${ }^{\mathrm{e}}$ siècle, la biologisation de la différence des sexes justifie le fait de placer les fonctions reproductives des femmes au centre de leur vie et de la sphère privée.

La modernité est donc marquée par ce que Wieviorka appelle le « principe de démarcation » $(1997$, p. 21$)$ entre le public et le privé, donnant ainsi lieu à la « double équation » (id., p. 23) qui 
consiste à associer la sphère privée aux femmes et la sphère publique aux hommes.

À partir des années 1970, les critiques féministes ont impulsé une redéfinition de la séparation entre ces deux sphères, montrant que celle-ci est source d'inégalités entre hommes et femmes, en particulier dans la vie professionnelle, familiale et politique. Les recherches féministes ont également mis en évidence les diverses significations de la dichotomie public / privé, notamment les deux ambiguïtés qui la caractérisent.

La première ambiguïté réside dans le fait que la distinction public / privé se réfère non seulement à la distinction entre l'État et la société, mais aussi à la distinction entre vie non domestique et vie domestique. Ce que Susan Okin appelle « le domaine socioéconomique intermédiaire » — c'est-à-dire, ici, la société et la vie non domestique - correspond au domaine privé dans le premier cas, et au domaine public dans le second. Les courants dominants de la théorie politique font généralement l'économie d'une analyse de cette ambiguïé, adoptant implicitement l'une des deux définitions et négligeant le plus souvent la sphère domestique. Selon Carole Pateman, la distinction entre État et société n'est qu'une "scission de la sphère publique en deux sous-sphères", où la vie domestique "occupe une position extrêmement ambiguë» (2000, p. 112). Ceci a amené Georgina Waylen à parler des "dangers d'une dichotomisation exagérée de la division entre l'État et la société civile » (2000, p. 226). Ne voir le rapport public / privé qu'en termes de dichotomie et ne pas accorder d'importance à la sphère domestique signifie négliger non seulement la famille, mais aussi les rapports de force existant au sein de celle-ci. Comme le souligne Okin, les théoriciens politiques des courants dominants ignorent ainsi «la nature politique de la famille» et «l'importance de la justice dans la vie personnelle » (1991, p. 71).

La seconde ambiguïté concerne la dichotomie public / domestique, dont l'élément central est le caractère patriarcal de la division sexuée du travail. Selon la naturalisation de la différence des sexes, les femmes ont été jugées comme étant subordonnées aux hommes au sein de la famille. Ainsi, depuis le $\mathrm{XVII}^{\mathrm{e}}$ siècle, les droits protégeant les « individus » dans leur vie 
privée ne s'appliquaient souvent qu'aux hommes. Alors qu'aux $\mathrm{XVII}^{\mathrm{e}}, \mathrm{XVIII}^{\mathrm{e}}$ et $\mathrm{XIX}^{\mathrm{e}}$ siècles, des théoriciens politiques tels que Locke, Rousseau et Hegel séparent sphère publique de sphère privée et justifient la domination masculine dans la sphère domestique, la plupart des théoriciens actuels continuent de prôner la séparation des deux sphères, mais négligent les structures de pouvoir à l'intérieur de la famille, ainsi que la division sexuée du travail en tant que problème de justice sociale. Dans la mesure où le droit à la vie privée dans la sphère domestique découle de la nature patriarcale du libéralisme, la question est de savoir de quel droit à la vie privée et à l'intimité nous parlons. En d'autres termes, il faut se demander - dans la perspective de genre où commence et où cesse ce droit, à qui il s'applique et qui en décide. En outre, en cas de conflit ou d'inégalités dans la sphère domestique, dans quelle mesure l'idée libérale de non-intervention de l'État est-elle acceptable ? Existe-t-il des droits inviolables du domaine privé de la liberté sur lesquels l'État ne doit pas intervenir? (Dietz 2000, p. 129).

À l'époque où Locke en appelle au droit à la vie privée dans la Lettre sur la tolérance, les hommes ont alors le droit légal de battre leurs enfants et leurs femmes, ainsi que d'obliger celles-ci à avoir des rapports sexuels dans le mariage. Le droit individuel et libéral à la vie privée ne s'applique donc qu'aux hommes. Locke refuse aux femmes le statut d' "individu libre et égal»" (Pateman 2000, p. 107). La conception du droit à la vie privée est de nature patriarcale jusqu'à l'époque contemporaine. En Grande-Bretagne, il n'est illégal de battre sa femme que depuis 1962, et les relations sexuelles forcées dans le mariage ne sont reconnues comme des viols que depuis la fin des années 1990. Pourtant, encore de nos jours, certains pays occidentaux ne considèrent pas le viol dans le couple comme un crime (Wolf 2002).

Grâce aux revendications féministes et des défenseurs des droits des mineurs, le droit à la vie privée s'est progressivement développé pour inclure les droits individuels des femmes et des enfants. Parler de droit à la vie privée dans la sphère domestique et de séparation entre public et privé n'est donc possible que s'il existe une égalité de fait entre les membres d'une même famille. Néanmoins, les femmes et les enfants des deux sexes sont encore nombreux à être menacés ou victimes de violences domestiques. 
L'importance de prendre en considération la catégorie « famille » résulte du fait que, comme le signale Sylvia Walby (2000) - et contrairement à la conceptualisation de Bryan Turner qui en 1990, dans sa typologie de la citoyenneté, associe l'espace « privé » à l'autonomie et à la liberté de l'individu —, la famille n'est pas un espace autonome et libre dans la mesure où chaque membre a des intérêts et des pouvoirs différents au sein de celle-ci.

Ainsi, au lieu de retenir la dichotomie privé / public qui prête encore aujourd'hui à confusion, j'adopterai, à l'instar de Nira Yuval-Davis, le continuum État / société civile / famille afin d'analyser ce qui détermine les droits civils des individus, c'està-dire les droits relatifs à la protection contre la violence.

En effet, selon Barbara Brook, l'engagement à une séparation entre société civile et sphère domestique se trouve au centre de la démocratie libérale (Brook 1999, p. 93). L'État a bien un rôle déterminant dans la construction des frontières entre le public et le privé, donc également dans la structuration des rapports entre hommes et femmes dans la sphère domestique. Néanmoins, cet engagement à une distinction entre les deux sphères n'est qu'une abstraction et la non-intervention a servi à masquer la façon dont l'État a façonné le genre et la famille (Brook 1999, p. 93).

Tout comme la séparation entre sphère privée et publique, la notion de sphères égales mais séparées n'est pas naturelle et dépend du niveau d'intervention de l'État. Brook rappelle que la notion "égales mais séparées 》 est liée au fait que la légitimité de l'État est basée sur l'idée de contrat social. En effet, le consentement de personnes qui sont égales est à la base de la notion de contrat (Brook 1999, p. 94). Or, dans The Sexual Contract (1988), Carole Pateman a montré comment le « contrat social » masque la manière dont il est basé sur le maintien du pouvoir masculin et sur la domination du corps des femmes. En d'autre termes, selon Brook, la séparation privé / public et le contrat social sont fondés sur l'accès des hommes au corps des femmes (Brook 1999, p. 94).

Cela signifie-t-il que la suppression de la séparation privé / public garantisse une réelle amélioration des droits individuels des femmes? Des principales revendications féministes remettant en cause la division privé / public, il a été dit — notamment 
par Mason, Sharp et Thorton - qu'elles comportent le risque de renforcer le pouvoir du public sur le privé, car le public a tendance à tirer vers lui le privé. Par exemple, placer l'avortement dans la sphère publique peut renforcer le pouvoir de l'État de prendre des décisions qui sont contraires aux choix de certaines femmes (Brook 1999, p. 95). Selon Brook, la régulation par l'État du corps des femmes enceintes permet de s'interroger sur la manière dont certains corps ont fait l'objet de davantage de contrôle que d'autres au regard de la loi (ibid.).

\section{La distinction privé / public face}

\section{à la différence culturelle liée au genre}

Ceci m'amène à aborder le thème de la différence culturelle et la manière dont elle s'articule à la division privé / public et à la dimension de genre. Tant au niveau académique que politique, le multiculturalisme britannique cherche actuellement à défendre la diversité culturelle, à promouvoir l'égalité des chances et à combattre les discriminations raciales. Cet idéal de la diversité - aussi appelé «reconnaissance de la différence » par Nancy Fraser $(1997$, p. 11) — succède à celui de la politique de la solidarité mise en œuvre avant les années 1970 à travers la promotion de l'État-providence. Solidarité et diversité peuvent être complémentaires. Néanmoins, selon Alan Wolfe et Jytte Klausen, seul le partage des mêmes valeurs et styles de vie facilite la solidarité (Wolfe, Klausen 2000, p. 28). Solidarité et diversité sont-elles compatibles face à un multiculturalisme britannique qui, en tant que catégorie d'analyse, se décline surtout en termes de $"$ race ${ }^{1}$ ? Ce multiculturalisme est racialisé puisque la défense et la promotion des groupes ethniques en GrandeBretagne passent principalement par des politiques et des législations dites « antiracistes ».

Face à un multiculturalisme qui se concentre sur la différence culturelle portant sur la « race », quelle doit être la position de

\footnotetext{
${ }^{1}$ Les politiques et législations « antiracistes » et «multiculturalistes » ont été mises en place par les différents gouvernements britanniques depuis les années 1960. Elles représentent les deux grandes approches idéologiques qui constituent $l$ '« industrie des relations raciales ».
} 
l'État vis-à-vis de certains particularismes comme par exemple la polygamie, le mariage forcé ou l'excision, dont la différence culturelle porte principalement sur le « sexe » socialement construit ? Par ailleurs, quel sens prend la notion de droits individuels des femmes issues de groupes ethniques, dès lors que cellesci mettent en avant la dimension « raciale» de leur différence culturelle et préfèrent suivre les traditions du groupe même si ces dernières vont à l'encontre de l'intégrité du corps des femmes?

Au continuum État / société civile / famille que propose Nira Yuval-Davis, j'ajouterai, dans un contexte multiculturel, la composante " groupe ethnique " entre la société civile et la famille, tout en considérant que ces sphères, comme le souligne YuvalDavis, ne sont jamais homogènes, et que les différentes composantes de l'État peuvent se contredire et avoir des effets multiples sur le genre, la classe et la « race» (Yuval-Davis 1998, p. 82).

Par ailleurs, même si les valeurs de la famille sont souvent le reflet de celles du groupe ethnique, il arrive que des conflits liés au genre opposent la famille et le groupe ethnique, terme que je définis d'après les travaux d'Immanuel Wallerstein et de Fredrik Barth. Pour Wallerstein, il s'agit d'un des « modes de construction de la notion de peuple » (1997, p. 106), et d'un terme qui en est venu à remplacer celui de « minorité » (id., p. 104). Selon lui, le groupe ethnique est « une catégorie culturelle, définie par certains comportements persistants, transmis de génération en génération » (id., p. 105), et selon Barth, « une forme d'organisation sociale » dont la caractéristique principale est «l'auto-attribution ou l'attribution par d'autres à une catégorie ethnique » (1969).

Les critiques féministes de la séparation privé / public se sont également appliquées au domaine de la différence culturelle, créant ainsi des tensions entre féminisme et multiculturalisme. Dans la mesure où la dichotomie privé / public permet la différence culturelle, il faut se demander jusqu'où on peut aller dans la reconnaissance et la protection des traditions culturelles des groupes, et à partir de quel moment un particularisme, qui s'avère dangereux pour l'intégrité des femmes, doit relever du domaine de la responsabilité de l'État ou au contraire de la sphère privée (Bano 2000, p. 3). 
Les recherches féministes ont mis en évidence l'absence de la dimension de genre des principaux courants de pensée sur la diversité culturelle, et en particulier sur la compatibilité entre diversité et égalité. Je prendrai comme exemple deux théoriciens du multiculturalisme britannique, John Rex et Bhikhu Parekh.

John Rex ne résout pas le problème de l'inégalité entre hommes et femmes lorsqu'il propose que la différence culturelle continue à relever du domaine privé. Son idée de multiculturalisme risque au contraire de renforcer les inégalités hommes-femmes. Tel est le cas quand il souhaite qu'une liberté de contrôle soit accordée au groupe ethnique pour ce qui est de la résolution des conflits de valeur dans les affaires familiales, ou quand il propose que le groupe ethnique soit subventionné pour prendre soin de ses membres. Face à cette vision homogénéisante du groupe et à la relégation de la différence culturelle dans la sphère privée, l'idée de Rex de promouvoir l'égalité des chances dans le domaine public ${ }^{2}$ semble peu efficace pour résoudre le problème de l'oppression des femmes au sein de leur propre groupe ethnique. Enfin, l'analyse de Rex présente le danger de ne concerner que la « race», l'ethnicité et les structures de classe ${ }^{3}$ (Rex 1986, p. 121-135).

Bhikhu Parekh critique le fait que la culture, la langue et la religion de la société d'accueil soient célébrées dans la sphère publique, ce qui relègue injustement les identités culturelles, ethniques et religieuses des «minorités » à la sphère privée. Il prône donc la promotion des groupes ethniques et religieux dans la sphère publique, tout en défendant la non-racialisation des groupes ethniques, la vision du groupe en tant qu'ensemble d'individus, et le partage de valeurs communes afin de fixer les limites du tolérable dans la différence culturelle (Parekh 2001, p. 693-695). L'objectif de ce modèle multiculturaliste est que les minorités ethniques aient le pouvoir de changer la société, et que l'interaction entre minorités et société dominante parvienne à redéfinir l'identité britannique (Britishness). Placer la défense

\footnotetext{
${ }^{2}$ Pour lui, les premières institutions constituant le domaine public sont les lois, la politique et l'économie (Rex 1986, p. 123).

${ }^{3}$ Rex n'élude pas les critiques féministes du multiculturalisme, néanmoins il ne prend pas position (Rex 1986, p. 127).
} 
de l'identité ethnique et religieuse dans la sphère publique est certes louable, mais seule l'identité collective est ici prise en compte. Or, garantir les différences culturelles dans le domaine public n'assure pas de fait la fin de la violence faite aux femmes au nom de la religion et des traditions.

Avant de se demander si la différence culturelle doit relever du domaine privé ou public, il faudrait partir des droits individuels des femmes face à la différence culturelle, c'est-à-dire s'interroger sur la manière dont cette différence affecte les femmes dans leur droit à la protection contre la violence.

Des féministes comme Susan Okin et Anne Phillips sont favorables à une démocratisation de la sphère privée, qui permettrait par là même la démocratisation de la sphère publique. Néanmoins, elles pensent qu'il est important de maintenir une distinction entre le privé et le public, car il doit y avoir un droit à l'intimité (privacy), c'est-à-dire un principe de libre choix. Ceci semble impossible dans le cas d'une pratique telle que l'excision qui, dans la majorité des cas et bien qu'elle ne comprenne pas d'intention criminelle, n'est pas une liberté puisqu'elle est imposée entre la naissance et l'adolescence. Pour reprendre les termes d'Okin (1991, p. 76), pour qu'il y ait démocratisation de la sphère privée, il est nécessaire que celle-ci soit non seulement compatible avec le respect du droit à l'intimité, mais aussi avec la sécurité physique des femmes et des enfants.

\section{Le cas de l'excision}

\section{Les populations exposées à l'excision en Grande-Bretagne}

Je m'arrêterai sur l'excision dans la mesure où elle est l'expression extrême du contrôle et de la domination sociale du corps des femmes. Selon la définition de l'Organisation mondiale de la santé (OMS) :

[L'excision est] l'extraction d'une partie ou de la totalité des organes génitaux externes féminins ou toute autre blessure des organes génitaux féminins pour des raisons culturelles ou non thérapeutiques (OMS 1997).

On distingue trois types d'excision: Les deux premiers consistent en l'ablation d'une partie ou de la totalité du clitoris, 
avec ou sans ablation des petites lèvres; le troisième, l'infibulation, est «l'excision d'une partie ou de la totalité des organes génitaux externes et la fermeture / rétrécissement de l'ouverture vaginale » (ibid.).

À l'heure actuelle, la Grande-Bretagne est le pays de l'Union européenne qui accueille le plus grand nombre de femmes provenant de sociétés qui pratiquent l'excision (Leye, Bruyn, Meuwese 1998 ; Osborn, Boseley 2000). En 1994, on estimait qu'entre 3000 et 4000 petites filles et jeunes femmes étaient excisées chaque année en Grande-Bretagne (Boot 1994, p. 2). Actuellement, l'association FORWARD (Foudation For Women's Health Research and Development) estime qu'elles sont un peu plus de $6000^{4}$ et qu'environ 10000 sont menacées de l'être. D'après les estimations les plus récentes de FORWARD (2001), les petites filles et jeunes femmes qui chaque année en GrandeBretagne sont exposées à l'excision ou sont déjà excisées sont, pour les moins de 16 ans, environ 6400 , et pour les plus de 16 ans, environ 74800 .

Les populations les plus exposées à cette pratique en GrandeBretagne sont originaires, pour les États membres du Commonwealth, des pays suivants: Ghana, Nigeria, Sierra Leone, Gambie, Kenya, Tanzanie et Uganda. Mais elles viennent aussi d'autres pays comme le Soudan, la Côte-d'Ivoire, le Togo, le Zaïre, l'Éthiopie et la Somalie. Ces populations vivent principalement dans les villes de Londres, Manchester, Liverpool, Sheffield, Birmingham, Leicester et Cardiff (Read 1999; All Party Parliamentary Group on Population, Development and Reproductive Health 2000). En Grande-Bretagne et essentiellement à Londres, la communauté somalienne est aujourd'hui la plus importante et la plus exposée à l'infibulation (Read 1999, p. 20), pratique qui touche $98 \%$ des femmes et des petites filles en Somalie (Williams 1999, p. 12).

Si l'on se place dans la perspective des «acteurs et actrices" issu(e)s de sociétés qui pratiquent l'excision, cette dernière est censée permettre à la jeune femme une affirmation de son identité sociale ; elle assurerait son propre " honneur », celui de sa famille et de sa communauté, en lui donnant accès au statut de

\footnotetext{
${ }^{4}$ Estimations non publiées et réalisées en mai 2001 à Londres.
} 
femme adulte, au mariage et à la maternité. En Grande-Bretagne, les familles qui font exciser leurs filles considèrent également l'excision comme un moyen d'empêcher que celles-ci ne se marient en dehors de leur groupe ethnique (Dorkenoo, Elworthy 1992/93). En outre, les jeunes filles sont d'autant plus fragilisées qu'elles sont victimes d'une double assignation dans le pays d'immigration. Elles sont en effet partagées entre, d'un côté, le respect de la tradition pour une raison de reproduction symbolique et sociale dont elles doivent assumer la responsabilité, et de l'autre, la laïcité, la modernité et l'« intégration ». Par ailleurs, les jeunes filles migrantes sont d'autant moins libres que se produit un durcissement des appartenances communautaires dans la société d'accueil. Comme l'explique Shamis Dirir :

Il est clair que le réseau de soutien et l'identification culturelle s'inscrivent toujours contre la culture britannique dominante (Dirir 1989, p. 3).

C'est ce que Michel Wieviorka appelle des «différences qui sont produites » dans la mesure où "des groupes et des personnes se construisent et se transforment dans leur spécificité culturelle ». Lors de ces processus, il arrive que « ce qui prime, ce [soit] le souci de se doter de racines [...] d'une vie communautaire qui permet de résister à l'universalisme d'une culture dominante arrogante », ainsi qu'à "l'expérience vécue du rejet social ou de la discrimination raciste » (Wieviorka 1997, p. 18). Ainsi, Shamis Dirir affirme que les «attitudes racistes » de la Grande-Bretagne font que les groupes ethniques se sentent «menacés », ce qui renforcerait les pressions culturelles pour continuer à pratiquer l'excision. Par « racisme », j'entends hostilité intergroupale renvoyant à des relations coloniales en vertu desquelles la « race» est, selon David Mason, «un rapport social caractérisé par une distribution inégale de pouvoir ».

Les actions sociales sont ordonnées, justifiées et expliquées par rapport aux systèmes de symboles et de croyances qui insistent sur l'importance sociale et culturelle de caractéristiques biologiquement fondées (Mason 1995, p. 9) ${ }^{5}$.

\footnotetext{
${ }^{5}$ Je n'adopte donc pas une définition «large » du racisme qui consiste à « englober sous le nom de 'racisme' [...] toutes les oppressions de minorités qui $[\ldots]$ conduisent à divers phénomènes de 'racisation' de groupes sociaux :
} 
Aux raisons qui font que l'excision perdure, il faut ajouter la crainte d'ostracisme de la part du groupe ethnique concerné et de la famille en cas de non-excision; la pression du groupe sur les mères chargées de l'opération; et le besoin de reproduire les traditions de la société d'origine dans la société d'accueil afin de maintenir des liens avec la communauté africaine et le pays d'origine.

L'absence d'un code des droits de la citoyenneté et la tradition britannique du droit jurisprudentiel (Common Law) ont permis de donner un espace aux valeurs culturelles et aux besoins des immigré(e)s (Stolcke 1995). Néanmoins, le multiculturalisme devrait poser le problème de ce qui est acceptable ou pas dans cette diversité culturelle. Selon les principes de justice défendus par l'État britannique, l'excision est une violation des droits fondamentaux de la personne à la vie, à la liberté et à l'intégrité physique et psychique (All Party Parliamentary Group on Population, Development and Reproductive Health 2000). Pour le système juridique britannique, il y a défense des particularismes privés tant que ces derniers ne sont pas en contradiction avec les lois nationales et les conventions internationales relatives aux droits de l'homme et de la femme ${ }^{6}$. Depuis 1985, la loi Prohibition of Female Circumcision Act interdit l'excision sur le sol britannique. De plus, la loi sur les droits de l'enfant (Children Act) de 1989, permet aux services municipaux :

[d'] exercer leurs pouvoirs s'ils ont des raisons de croire qu'une fillette est susceptible d'être ou d'avoir été soumise à l'excision.

Cette pratique est donc contraire au concept libéral de citoyenneté qui «affirme l'égalité juridique et politique d'individus inégaux » (Schnapper 1998, p. 13).

Dans ces conditions, comment doivent s'articuler citoyenneté, particularismes culturels et genre ? Stephen Castles a proposé une citoyenneté multiculturelle qui inclurait droits civils, politiques, sociaux et culturels (Castles 1994, p. 17). Bien que Castles

groupes ethniques, mais aussi femmes, déviants sexuels, malades mentaux, sous-prolétaires » (Balibar 1997, p. 70).

${ }^{6}$ La Grande-Bretagne est entre autres signataire de la Convention européenne sur l'exercice du droit des enfants (1996) et de la Convention de sauvegarde des droits de l'homme et des libertés fondamentales (1998). 
ait moins traité la dimension sexuée de la citoyenneté que sa dimension multiculturelle, il définit la citoyenneté multiculturelle comme un «système de droits et de libertés qui cependant sont limités par des exigences telles que [...] l'égalité des sexes» (Castles, id.). Dans le cas de l'excision, parler de citoyenneté multiculturelle signifie donc condamner et interdire cette pratique. Néanmoins, comme le souligne Martine Spensky :

L'État libéral se refuse à intervenir dans ce qui est construit comme la sphère privée puisqu'elle est censée représenter l'aire de liberté du citoyen. Toute intervention dans cette sphère est ressentie comme une menace pour la citoyenneté (Spensky 2001, p. 245).

\section{Genre, racialisation, ethnicité et politique d'appartenance : des femmes somaliennes contre l'État britannique}

Il est d'autant plus difficile d'intervenir contre l'excision que certaines femmes somaliennes du secteur non gouvernemental - qui sont pourtant fermement opposées à cette pratique ont, dans les années 1980, perçu la loi de 1985 comme « raciste », car elles voyaient dans l'interdiction de l'excision pour «des raisons de coutume et de rituel », une attaque à leur culture. Shamis Dirir - responsable somalienne de la campagne alors menée contre le gouvernement par son organisation domiciliée à Londres : la Somali Women's Association (SWA), appelée par la suite London Black Women's Health Action Project (LBWHAP), et aujourd'hui Black Women's Health and Family Support (BWHFS) - écrit en 1989 :

Les tentatives d'abolir la pratique sont souvent comprises (et peut-être est-ce réellement l'intention qui sous-tend la loi de 1985 sur l'excision) comme une tentative de s'en prendre aux valeurs traditionnelles (Dirir 1989, p. 33).

Fondée en 1979 dans le quartier de Tower Hamlets, la Somali Women's Association fait alors partie de la Somali London Community and Cultural Association (SLCCA) et est dirigée par Shamis Dirir. La présence somalienne à Londres date de la fin du XIX $^{\mathrm{e}}$ siècle, mais ce n'est qu'à partir de la fin des années 1970 que la communauté somalienne s'organise de manière institutionnelle, notamment dans l'est de Londres. Des femmes de la ville d'Aden (capitale du Yémen du sud), arrivées à Londres 
dans les années 1960, sont à l'origine de la création de la SLCCA. C'est la première association somalienne qui se forme dans l'est de Londres. La création de la Somali Women's Association s'inscrit dans l'histoire du mouvement britannique pour la santé des femmes de la fin des années 1970 et des années 1980. Ce mouvement fait émerger des projets promouvant la santé, à l'initiative de femmes issues de groupes ethniques. La SWA est principalement dirigée par des Somaliennes elles-mêmes excisées et qui œuvrent, depuis le début des années 1980, contre l'excision et l'infibulation, à Londres et dans le reste de la Grande-Bretagne. Parmi les associations domiciliées à Londres et travaillant pour la prévention de ces pratiques, cette association était et continue d'être, à mon avis, la plus importante pour ce qui est de sa proximité avec la communauté somalienne.

En 1985, la déclaration adoptée en assemblée générale annuelle ${ }^{7}$ de cette organisation, explique son opposition à l'interdiction d'excision pour raisons de « coutume et rituel» et non pour des raisons «physiques et mentales » :

[Nous sommes] totalement opposés à cette loi car elle opère une distinction entre l'excision pour raisons de santé mentale et physique, et l'excision liée à la coutume et aux rites. Il s'agit à l'évidence d'une approche raciste dans la mesure où seules les femmes noires appartenant à une culture traditionnelle qui implique une telle pratique auraient recours à l'excision pour des raisons liées à "la coutume et aux rites ». S'il s'agit d'interdire l'excision ou d'autoriser certaines exceptions, de telles distinctions n'ont pas lieu d'être.

La position de cette organisation représente un défi pour l'articulation entre affirmation des spécificités culturelles et participation aux valeurs universelles. La reconnaissance de la différence se réfère au fait qu'un groupe ethnique revendique des spécificités culturelles qui lui sont propres afin de continuer à vivre selon elles. Or, la position de ces femmes sur une possible loi contre l'excision ne correspond pas à une demande de reconnaissance dans le sens que je viens de donner, mais reflète une réaction à ce qui est ressenti comme une attaque à leur culture. Elles défendent l'individu contre l'excision au moyen

\footnotetext{
${ }^{7}$ Archives de la Black Women's Health and Family Support (BWHFS), Londres.
} 
de la revendication de leur identité et culture «noires ». Nous sommes ici dans la phase du processus de « racialisation» des groupes ethniques qui se construit à partir des individus subissant le racisme ou ce qu'ils perçoivent comme étant « raciste». Comme l'écrit Wieviorka :

Parfois, l'expérience vécue [...] de la discrimination raciste est décisive dans la construction d'une identité culturelle qui, à la limite, vient apporter sa réponse [...] à l'exclusion [...] en poussant l'acteur à s'approprier le discours de la différence qui lui était jusque-là surtout imposé du dehors (1997, p. 18-19).

Par ailleurs, pour ce qui est de la défense de la culture du groupe par rapport aux droits de l'individu, comme le souligne Claude Dubar, il peut y avoir rupture avec les valeurs traditionnelles qui traduisent une domination des femmes par les hommes. Comme cela est le cas pour cette association de femmes somaliennes, cette rupture — qui est basée sur la "coordination d'intérêts motivés rationnellement» ou "formes sociétaires», et non sur les "formes communautaires ${ }^{8}$ — se fait même parfois à travers la promotion du groupe d'origine (Dubar 2000, p. 191).

Ici, la question est de savoir jusqu'à quel point le besoin d'application du principe de citoyenneté est compatible avec les revendications d'un groupe ethnique qui craint une stigmatisation de celui-ci en cas de promulgation d'une loi. La position des femmes de la Somali Women's Association pouvait être certes compréhensible du point de vue de l'évolution interne des membres du groupe ethnique par rapport à leur attitude envers l'excision et envers la société d'accueil, mais elle était en contradiction avec l'obligation urgente qu'avait alors la Grande-Bretagne d'assurer la défense des droits des individus. Néanmoins, la loi de 1985 n'a jamais été appliquée malgré le nombre croissant d'excisions sur le sol britannique et, comme le rappelle Wieviorka, "les dimensions identitaires de l'ethnicité [...] ne sont pas favorables à la prise en compte des femmes comme sujets de leur propre existence, surtout lorsque leur

\footnotetext{
${ }^{8}$ Max Weber distingue les «formes sociétaires » et les «formes communautaires ». Celles-ci dépendent du «sentiment subjectif d'appartenir à une même collectivité » et de la « force de la tradition » (Dubar 2000, p. 29).
} 
principe d'identité tend au communautarisme » (Wieviorka 1997, p. 25). De quelle protection des droits individuels des femmes parle-t-on alors? En théorie, l'État britannique a une conception libérale des droits qui place l'individu avant le groupe. Néanmoins, dans la pratique, les attitudes multiculturalistes priment sur la loi et, concrètement, les services sociaux et la police n'osent pas intervenir contre l'excision de peur d'être accusés de «racisme». Or, comme l'a souligné Pragna Patel (2002), on sait l'« effet dévastateur » des politiques et des perceptions multiculturalistes «sur la reproduction du pouvoir patriarcal au sein des minorités ethniques ». Dans la pratique, on a donc une conception communautariste des droits qui envisage l'individu par rapport à son appartenance au groupe, qui est racialisante et fonctionne aux dépens des droits des femmes. En outre, les personnes qui, en Grande-Bretagne, militent contre l'excision, reprochent au gouvernement son absence d'engagement financier vis-à-vis des associations africaines domiciliées à Londres (Mwangi-Powell 1999, p. 26). Elles lui reprochent de ne pas entreprendre de recherche sur la prévalence de l'excision. Selon Shamis Dirir, interviewée en 2001 :

Il faudra [...] beaucoup de temps pour arrêter l'excision. Le gouvernement dit qu'il faut l'éradiquer, mais ne nous donne pas les moyens d'y parvenir. L'excision continue à se pratiquer dans ce pays. Si le gouvernement veut faire quelque chose, il devrait le faire vite (Gillan 2001, p. 3).

Enfin, quelle est la valeur accordée par les groupes ethniques concernés à un État britannique qui a voulu définir, au moyen d'une loi considérée par certain(e)s comme " raciste », les frontières des droits civils des femmes et des petites filles exposées à l'excision? Quel sens prend alors la politisation du privé lorsque l'État intervient contre des spécificités culturelles sans pour autant réussir à protéger les femmes, puisque la tradition perdure au sein des groupes ethniques concernés ?

Les revendications des femmes somaliennes de cette association se situent principalement dans la sphère publique. En effet, à travers leur activité associative de prévention de l'excision, elles participent aux principes de la démocratie moderne ou, comme le note Chantal Mouffe, à la citoyenneté en tant qu'«identité politique commune de personnes [...] unies par leur identifica- 
tion partagée à une interprétation donnée d'un ensemble de valeurs éthico-politiques (Mouffe 2000, p. 187).

Néanmoins, les revendications de ces femmes semblent aussi s'inscrire dans ce que Yuval-Davis appelle « la politique d'appartenance ». Il ne s'agit pas d'appartenance au sens de membership, ni de droits et de devoirs, mais d'émotions. L'appartenance, belonging, ne se réduit pas pour autant aux identités et aux identifications. Selon Yuval-Davis, il s'agit d'un «besoin émotionnel profond des gens qui se forme dans l'enfance » et qui peut même perdurer lors d'abus au sein de la famille. Pour elle, on ne peut pas ne pas prendre en compte le « côté émotionnel » dans nos théorisations de la justice sociale. Dans un contexte de migration, l'identité ethnique se construit et se reconstruit de manière défensive :

Notre propre rapport à la communauté, au pays d'origine et à l'État s'articule dès lors que la sûreté et la stabilité de ce rapport sont menacées. Dans ce cas, les récits narratifs individuels, collectifs et institutionnels d'appartenance se politisent (YuvalDavis 2003).

Selon Yuval-Davis, la politique d'appartenance des femmes est paradoxale :

Les femmes sont vues comme les signifiants de l'honneur collectif [...] mais elles sont également l'Autre au sein du groupe, car soumises à différentes formes de contrôle au nom de la " culture et de la tradition » (Yuval-Davis 2003).

Ceci amène également au paradoxe auquel s'est intéressé la recherche féministe et que j'ai évoqué auparavant, à savoir celui qui porte sur les relations, pour les femmes, entre l'appartenance et la sécurité, car le danger - pour celles-ci — se trouve souvent là où se développent leurs liens d'appartenance. Il s'agit de ce que les féministes appellent «l'ennemi de l'intérieur », indiquant que ce sont souvent les membres les plus proches qui sont les plus violents. Il n'y a pas d' " ennemi » à proprement parler dans le cas de l'excision, mais les liens d'appartenance sont une condition à la réalisation de cette pratique.

La réaction des femmes de la Somali Women's Association reflète le paradoxe de leur appartenance en tant que responsables de la reproduction symbolique du groupe, et en tant 
qu'Autre que l'on contrôle, mais qui tente aussi d'abolir une pratique qui va contre les femmes. Face aux difficultés d'une transformation radicale des rapports sociaux de sexe à l'intérieur du groupe et de la famille, elles tendent, malgré leur opposition à l'excision, à défendre la culture du groupe face au gouvernement du pays d'accueil. De même, le fait que l'excision soit toujours pratiquée en Grande-Bretagne malgré une loi interdisant cette pratique, montre que les liens d'appartenance prévalent sur le concept de citoyenneté.

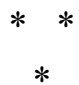

En vue de concilier universalisme et particularisme, Nancy Fraser propose d'établir des formes multiculturelles publiques afin de reconnaitre les multiples façons d'exister en tant qu'être humain (Fraser 1997, p. 183-184). Pour Nira Yuval-Davis, il s'agit d'atteindre "l'universalité dans la diversité ». Néanmoins, seule l'étude de l'écart entre les discours et les pratiques permet d'envisager la manière dont il serait possible de concilier particularismes et exercice de la citoyenneté civile par les femmes victimes de pratiques les privant de liberté. Dans le cas de l'excision, tant que les liens d'appartenance auront la prééminence sur la pratique citoyenne, l'intervention du public dans le privé par la seule voie législative ne sera que limitée et insuffisante. Comme le soutient Nahid Toubia (Toubia, Izett 1999), chirurgienne soudanaise, fondatrice et présidente de l'organisation RAINBO (Research, Action and Information Network for the Bodily Integrity of Women), seul un changement social à long terme peut faire progressivement reculer l'excision. Néanmoins, la défense des droits des femmes estelle possible là où existe, pour reprendre les termes de Foucault, un pouvoir qui s'exerce à partir d'un «jeu de relations inégalitaires » (Foucault 1976, p. 123) entre l'État et la société civile ? Tant qu'il ne s'engagera pas à fournir aux associations travaillant contre cette pratique les moyens réels permettant de mettre en œuvre un changement social, et tant que les pouvoirs locaux hésiteront à intervenir, l'État continuera d'apparaître comme un instrument de domination patriarcale et se montrera 
incapable de protéger les femmes comme il le doit dans l'exercice de leur citoyenneté civile.

\section{Références}

All Party Parliamentary Group on Population, Development and Reproductive Health (2000). Report of the Parliamentary Hearings on Female Genital Mutilation Held on 23 and 24 May 2000. London, All Party Parliamentary Group on Population, Development and Reproductive Health.

Balibar Étienne (1997). « Racisme et nationalisme ». In Balibar Étienne, Wallerstein Immanuel (eds). Race, nation, classe : les identités ambiguës. Paris, La Découverte.

Bano Samia (2000). "Muslim South Asian Women and Customary Law in Britain ». Journal of South Pacific Law, vol. 4, $\mathrm{n}^{\circ} 6$.

Barth Fredrik (1969). Ethnic Groups and Boundaries. The Social Organization of Culture Difference. Bergen, Oslo, Universitet Forlaget [trad. in Poutignat Philippe, Streiff-Fenart Jocelyne (eds) (1995). Théories de l'ethnicité. Paris, PUF « Le sociologue »].

Boot Jayshree (1994). Female Genital Mutilation: Implications for the London Borough of Waltham Forest. Social Justice Committee, Chief Executive's / Women's Unit, London Borough of Waltham Forest, November.

Brook Barbara (1999). Feminist Perspectives on the Body. New York, Longman.

Castles Stephen (1994). « Democracy and Multiculturalism Citizenship. Australian Debates and their Relevance for Western Europe ». In Bauböck Reiner (ed). From Aliens to Citizens. Avesbury, Aldershot.

Dietz Mary (2000). « Tout est dans le contexte : féminisme et théories de la citoyenneté ». In Ballmer-Cao Thanh Huyê'n, Mottier Véronique, Sgier Lea (eds). Genre et politique. Débats et perspectives. Paris, Gallimard.

Dirir Shamis (1989). Silent Tears. London, London Black Women's Health Action Project.

Dorkenoo Efua, Elworthy Scilla (1992/93). Female Genital Mutilation: Proposals for Change. London, Minority Rights Group Int.

Dubar Claude (2000). La crise des identités. L'interprétation d'une mutation. Paris, PUF « Le lien social».

Foucault Michel (1976). Histoire de la sexualité. 1. La volonté de savoir. Paris, Gallimard. 
Fraser Nancy (1997). Justice Interruptus: Critical Reflections on the «Postsocialist » Condition. New York, Routledge.

Gillan Audrey (2001). «Bringing Female Genital Mutilation into the Light ». Sohu, Guardian News Service, January.

Leye Els, Bruyn (de ) Maria, Meuwese Stan (1998). Proceedings of the Expert Meeting on Female Genital Mutilation. Ghent (Belgique), November 5-7.

Mason David (1995). Race and Ethnicity in Modern Britain. Oxford, Oxford University Press.

Mouffe Chantal (2000). «Féminisme, citoyenneté et démocratie plurielle ». In Ballmer-Cao Thanh Huyê'n, Mottier Véronique, Sgier Lea (eds). Genre et politique. Débats et perspectives. Paris, Gallimard. Mwangi-Powell Faith (1999). « Developing Educational Materials for the Community and for those Working with them ». In Moving Forward. Report of the Conference on FGM. London, Forward.

Okin Susan Moller (1991). "Gender, the Public and the Private ». In Held David (ed). Political Theory Today. Stanford, Calif., Stanford University Press.

OMS (1997). Female Genital Mutilation: A Joint WHO / UNICEF / UNFPA Statement. Genève, Organisation mondiale de la santé.

Osborn Andrew, Boseley Sarah (2000). «EU May Ban Aid to States that Allow Female Circumcision ». The Guardian, 30 November.

Parekh Bhikhu (2001). «The Future of Multi-Ethnic Britain. Reporting on a Report ». The Round Table, $\mathrm{n}^{\circ} 362$.

Patel Pragna (2002). « Back to the Future. Avoiding déjà vu in Resisting Racism ». In Anthias Floya, Lloyd Cathie (eds). Rethinking AntiRacisms. From Theory to Practice. London, Routledge.

Pateman Carole (1988). The Sexual Contract. Stanford, Calif., Stanford University Press.

— (2000). «Féminisme et démocratie ». In Ballmer-Cao Thanh Huyê'n, Mottier Véronique, Sgier Lea (eds). Genre et politique. Débats et perspectives. Paris, Gallimard.

Perrot Michelle (1998). Les femmes ou les silences de l'Histoire. Paris, Flammarion.

Read Deborah (1999). Out of Sight, Out of Mind? The Report of a Survey into Inter-Agency Policies and Procedures Relating to FGM in England and Wales. London, Forward.

Rex John (1986). Race and Ethnicity. Milton Keynes, Open University Press.

Schnapper Dominique (1998). La relation à l'Autre. Paris, Gallimard. 
Spensky Martine (2001). « Citoyenneté : droits des individus et droits des groupes ». Cahiers du genre, $\mathrm{n}^{\circ} 30$.

Stolcke Verena (1995). « Talking Culture. New Boundaries, New Rhetorics of Exclusion in Europe ». Current Anthropology, vol. 36, $\mathrm{n}^{\circ} 1$.

Toubia Nahid, Izett Susan (1999). Learning About Social Change: A Research and Evaluation Guidebook Using FC/FGM as a Case Study. New York, RAINBO.

Walby Sylvia (2000). « La citoyenneté est-elle sexuée ? » In BallmerCao Thanh Huyê'n, Mottier Véronique, Sgier Lea (eds). Genre et politique. Débats et perspectives. Paris, Gallimard.

Wallerstein Immanuel (1997). «La construction des peuples : racisme, nationalisme, ethnicité ». In Balibar Étienne, Wallerstein Immanuel (eds). Race, nation, classe : les identités ambiguës. Paris, La Découverte.

Waylen Georgina (2000). «Le genre, le féminisme et l'État : un survol ». In Ballmer-Cao Thanh Huyê'n, Mottier Véronique, Sgier Lea (eds). Genre et politique. Débats et perspectives. Paris, Gallimard.

Wieviorka Michel (1997). «Culture, société et démocratie ». In Wieviorka Michel (ed). Une société fragmentée ? Le multiculturalisme en débat. Paris, La Découverte.

Williams Linda (1999). « Experiences, Attitudes and Beliefs of Young Single Somalis Living in London ». Moving Forward. Report of the Conference on Female Genital Mutilation. London, Forward.

Wolf Sonia (2002). «Violence conjugale : une femme est tuée chaque semaine par son conjoint en Europe ». Agence France-Presse, Strasbourg, septembre.

Wolfe Alan, Jytte Klausen (2000). « Other People ». Prospect, December. Yuval Davis Nira (1998). Gender and Nation. London, Sage Publications. - (2003). " 'Human Security' and the Gendered Politics of Belonging ». Présenté au Symposium Justice, Equality and Dependency in the «Postsocialist » Condition. University of Warwick (Grande-Bretagne), March :

www2.warwick.ac.uk/fac/soc/sociology/gender/events/symposium/yuval/ 